\title{
Distinguishing Black Hole Microstates using Holevo Information
}

\author{
Wu-zhong Guo, ${ }^{1, \$}$ Feng-Li Lin, ${ }^{2, *}$ and Jiaju Zhang ${ }^{3,4, \dagger}$ \\ ${ }^{1}$ Physics Division, National Center for Theoretical Sciences, National Tsing Hua University, \\ No. 101, Sec. II, Kuang Fu Road, Hsinchu 30013, Taiwan \\ ${ }^{2}$ Department of Physics, National Taiwan Normal University, \\ No. 88, Sec. IV, Ting-Chou Road, Taipei 11677, Taiwan \\ ${ }^{3}$ Dipartimento di Fisica G. Occhialini, Università degli Studi di Milano-Bicocca, \\ Piazza della Scienza 3, 20126 Milano, Italy \\ ${ }^{4}$ INFN, Sezione di Milano-Bicocca, Piazza della Scienza 3, 20126 Milano, Italy
}

(Received 5 September 2018; published 21 December 2018)

\begin{abstract}
We use Holevo information in the two-dimensional conformal field theory (CFT) with a large central charge $c$ to distinguish microstates from the underlying thermal state. Holographically, the CFT microstates of a thermal state are dual to black hole microstate geometries in anti-de Sitter space. It was found recently that holographic Holevo information shows plateau behaviors at both short and long interval regions. This indicates that the black hole microstates are indistinguishable from the thermal state by measuring over a small region, and perfectly distinguishable over a region with its size comparable to the whole system. In this Letter, we demonstrate that the plateaus are lifted by including the $1 / c$ corrections from both the vacuum and nonvacuum conformal families of CFT in either the canonical ensemble or microcanonical ensemble thermal state. Our results imply that the aforementioned indistinguishability and distinguishability of black hole microstate geometries from the underlying black hole are spoiled by higher order Newton constant $G_{N}$ corrections of quantum gravity.
\end{abstract}

DOI: $10.1103 /$ PhysRevLett.121.251603

Introduction.-The black hole information paradox lies in the fact that a pure state seems to evolve into a thermal state through Hawking radiation, and thus it violates the unitarity of quantum mechanics. This paradox can be partially resolved if there exists black hole microstates, which are pure states that cannot be distinguished from the underlying thermal state. This resolution however calls for a complete theory of quantum gravity, which is beyond the reach at this moment. However, with the help of the anti-de Sitter/conformal field theory (AdS/CFT) correspondence [1] one may glimpse the answer for this quantum gravity problem from the viewpoint of its dual CFT.

Recently, it was proposed in [2] to characterize the distinguishability of the black hole microstates from its underlying thermal state by Holevo information. One can call it, in short, the distinguishability of black hole microstates. The thermal state of the whole system is described by

$$
\rho=\sum_{i} p_{i} \rho_{i}, \quad \rho_{i}=|i\rangle\langle i|,
$$

Published by the American Physical Society under the terms of the Creative Commons Attribution 4.0 International license. Further distribution of this work must maintain attribution to the author(s) and the published article's title, journal citation, and DOI. Funded by SCOAP ${ }^{3}$. with the orthonormal microstates $|i\rangle$ satisfying $\left\langle i \mid i^{\prime}\right\rangle=\delta_{i i^{\prime}}$. Note that $0 \leq p_{i} \leq 1, \sum_{i} p_{i}=1$. One would like to distinguish the microstates from the thermal state by performing measurements in a subsystem $A$, whose complement is denoted by $B$. The first step is to consider the relative entropy by comparing the reduced density matrix $\rho_{A, i}=\operatorname{tr}_{B} \rho_{i}$ of each of the microstates with the reduced density matrix $\rho_{A}=$ $\operatorname{tr}_{B} \rho$ of the corresponding thermal state, i.e.,

$$
S\left(\rho_{A, i} \| \rho_{A}\right)=\operatorname{tr}\left(\rho_{A, i} \log \rho_{A, i}\right)-\operatorname{tr}\left(\rho_{A, i} \log \rho_{A}\right) .
$$

This quantity is a well-defined divergence and characterizes the difference between the two reduced density matrices. The average relative entropy gives Holevo information

$$
\chi_{A}=\sum_{i} p_{i} S\left(\rho_{A, i} \| \rho_{A}\right)=S_{A}-\sum_{i} p_{i} S_{A, i},
$$

with entanglement entropies (EEs) $S_{A}=-\operatorname{tr}\left(\rho_{A} \log \rho_{A}\right)$, $S_{A, i}=-\operatorname{tr}\left(\rho_{A, i} \log \rho_{A, i}\right)$. It is the difference between the thermal state $\mathrm{EE}$ and the average $\mathrm{EE}$ of the microstates. Holevo information $\chi_{A}$ is the upper bound of the mutual information between the thermal state and any measurement inside $A$, which is aiming to reproduce the thermal state and to characterize the accessible information.

By construction

$$
0 \leq \chi_{A} \leq S_{\text {thermal }},
$$


with $S_{\text {thermal }}$ being the thermal entropy of the whole system:

$$
S_{\text {thermal }}=-\sum_{i} p_{i} \log p_{i}
$$

When $\chi_{A}=0, \rho_{A, i}=\rho_{A}$ so that the microstates are totally indistinguishable by measurements inside $A$. On the other hand, when $\chi_{A}=S_{\text {thermal }}, \rho_{A, i} \rho_{A, i^{\prime}}=0$ for arbitrary $i, i^{\prime}$ and thus the microstates are completely distinguishable.

To investigate the information loss paradox of black hole in Einstein gravity in the three-dimensional anti-de Sitter $\left(\mathrm{AdS}_{3}\right)$ background, i.e., the Bañados-Teitelboim-Zanelli (BTZ) black hole [3], we calculate Holevo information in a two-dimensional (2D) CFT. When the gravity is weakly coupled, the CFT has a large central charge [4]

$$
c=\frac{3 R}{2 G_{N}},
$$

with $G_{N}$ being the Newton constant and $R$ being the AdS radius. The $1 / c$ corrections on the CFT side correspond to quantum corrections on the gravity side.

We consider a 2D large $c$ CFT in thermal state on a cylinder with spatial period $L$. For an interval $A$ with length $\ell$, we denote Holevo information by $\chi(\ell)$. Holevo information $\chi(\ell)$ is monotonically increasing with respect to $\ell$. It is easy to see that

$$
\lim _{\ell \rightarrow 0} \chi(\ell)=0, \quad \lim _{\ell \rightarrow L} \chi(\ell)=S(L) .
$$

By using the holographic entanglement entropy (HEE) [5,6], it was recently found in Ref. [2] that holographic Holevo information shows plateau behaviors around both $\ell \rightarrow 0$ and $\ell \rightarrow L$. This indicates that the microstates are totally indistinguishable until the interval reaches a nonvanishing critical length, and are perfectly distinguishable after the interval reaches another critical length that is shorter than the length of the whole system. However, the HEE is only the classical gravity result, and it is expected that quantum corrections to the HEE [7-9] would resolve both plateaus of the holographic Holevo information. On the dual CFT side, these correspond to $1 / c$ corrections. The problem has been addressed in Ref. [10] for the 2D CFT due to the zero mass BTZ black hole. In this Letter, we consider the more general thermal states, including the canonical ensemble thermal state with both high and low temperatures, as well as the microcanonical ensemble thermal state. This is not only technically challenging by performing the thermal average over all eigenstates, i.e., including both primaries and their descendants, but also conceptually interesting to see if the peculiar nonthermal or nongeometrical descendants states found in Ref. [11] will be thermally averaged out so that the microstates remain almost ultralocally indistinguishable.
We find that Holevo information is not vanishing as long as the length of the interval is nonvanishing, and this indicates that the black hole microstates are distinguishable from thermal state as long as the measuring region is nonvanishing. We also find the Holevo information is smaller than the thermal entropy as long as the interval is shorter than the whole system.

For calculation convenience we choose that the interval $A$ is short, i.e., $\ell / L \ll 1$, and thus its complement $B$ has a length $L-\ell$ comparable to $L$. Then we have

$$
\begin{aligned}
& S_{A}=S(\ell), \quad S_{A, i}=S_{i}(\ell), \quad \chi_{A}=\chi(\ell), \\
& S_{B}=S(L-\ell), \quad S_{B, i}=S_{i}(L-\ell), \\
& \chi_{B}=\chi(L-\ell) .
\end{aligned}
$$

Note that $S_{A, i}=S_{B, i}$. To get the short and long interval Holevo information $\chi_{A}$ and $\chi_{B}$, we need to calculate the short and long interval EEs of thermal state, i.e., $S_{A}, S_{B}$, and the average of the short interval EEs of the microstates, i.e., $\sum_{i} p_{i} S_{A, i}$. For the short interval, as in Refs. [12-15], we use the operator product expansion (OPE) of twist operators [7,16-21] to calculate the short interval expansion of the EE. This method is still available for the long interval case [22-24].

Canonical ensemble thermal state with high temperature.-For a canonical ensemble thermal state we have

$$
p_{i}=\frac{\mathrm{e}^{-\beta E_{i}}}{Z(\beta)}, \quad Z(\beta)=\sum_{i} \mathrm{e}^{-\beta E_{i}}
$$

with $\beta$ being the inverse temperature. We consider high temperature limit $\beta / L \ll 1$ and omit the terms suppressed by the exponential factor $\mathrm{e}^{-2 \pi L / \beta}$. The thermal entropy is

$$
S(L)=\frac{\pi c L}{3 \beta},
$$

which is just the entropy of a nonrotating BTZ black hole. Using the HEE [5,6], one can get the holographic Holevo information [2]

$$
\chi_{\text {holo }}(\ell)=\left\{\begin{array}{cl}
0 & \ell<\frac{\beta}{2 \pi} \log 2 \\
\frac{\pi c L}{3 \beta} & \ell>L-\frac{\beta}{2 \pi} \log 2
\end{array} .\right.
$$

The holographic Holevo information $\chi_{\text {holo }}(\ell)$ with $(\beta / 2 \pi) \log 2<\ell<L-(\beta / 2 \pi) \log 2$ is unknown. The result is plotted in Fig. 1. There are plateaus at both $\ell<$ $(\beta / 2 \pi) \log 2$ and $\ell>L-(\beta / 2 \pi) \log 2$. We will resolve the plateaus in CFT.

We consider only contributions from the vacuum conformal family, and will briefly discuss the contributions from nonvacuum conformal families in the end of the Letter. For the short interval $A$ we have the EE [16] 

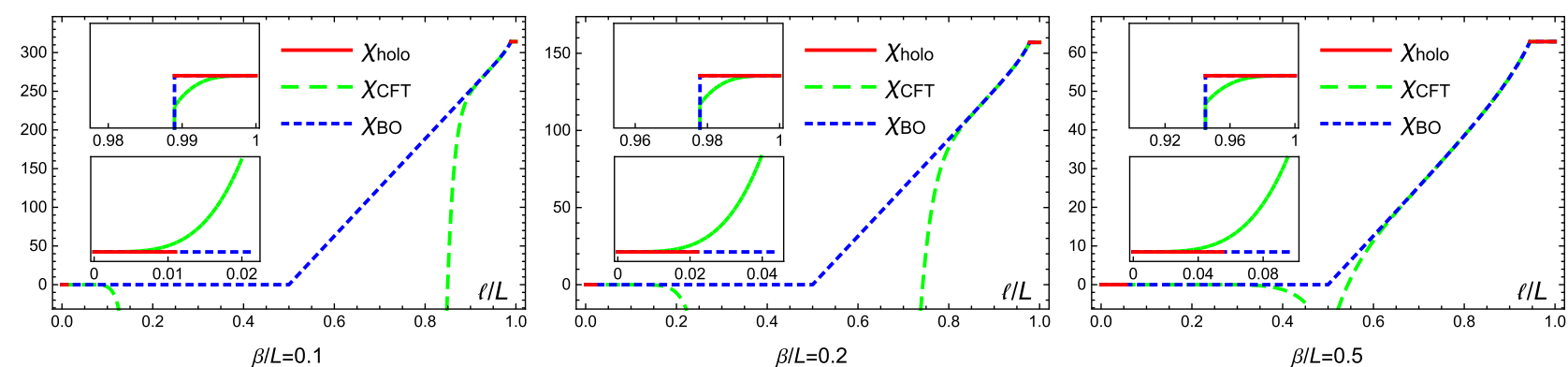

FIG. 1. The holographic Holevo information $\chi_{\text {holo }}$ [Eq. (11)], the short and long interval expansion of the CFT Holevo information $\chi_{\text {CFT }}$ [Eqs. (14) and (16)], i.e., (S14) and (S18) in the Supplemental Material [25], and the leading order $c$ Holevo information $\chi_{\text {BO }}$ [Eq. (18)], for the high temperature thermal state with $\beta / L=0.1$ (Left), $\beta / L=0.2$ (Middle), and $\beta / L=0.5$ (Right), respectively. The unknown region of holographic Holevo information $\chi_{\text {holo }}$ is left blank. To draw the figures we have set $c=30$.

$$
S_{A}=\frac{c}{3} \log \left(\frac{\beta}{\pi \epsilon} \sinh \frac{\pi \ell}{\beta}\right) .
$$

Though we do not calculate $S_{A, i}$ for all the pure states, using the results in Refs. $[15,26]$ we can get the average EE

$$
\begin{aligned}
\sum_{i} p_{i} S_{A, i}= & \frac{c}{3} \log \frac{\ell}{\epsilon}+\frac{\pi^{2} c \ell^{2}}{18 \beta^{2}}-\frac{\pi^{3} \ell^{4}(\pi c L+24 \beta)}{540 \beta^{4} L} \\
& +\frac{\pi^{4} \ell^{6}\left(\pi^{2} c^{2} L^{2}+72 \pi c \beta L+864 \beta^{2}\right)}{8505 c \beta^{6} L^{2}} \\
& +\cdots+O\left(\ell^{12}\right) .
\end{aligned}
$$

We have omitted some involved terms denoted by $\cdots$, and one can find full form of the equation in (S13) of the Supplemental Material [25]. There are technical issues in calculating the result to higher orders of $\ell$. See details in the Supplemental Material [25]. Combining them, we obtain the short interval Holevo information

$\chi_{A}=\frac{2 \pi^{3} \ell^{4}}{45 \beta^{3} L}-\frac{8 \pi^{4} \ell^{6}(\pi c L+12 \beta)}{945 c \beta^{5} L^{2}}+\cdots+O\left(\ell^{12}\right)$.

See full form of the equation in (S14) of the Supplemental Material [25]. We find that, to the order we consider, it is vanishing in the thermodynamic limit $[27,28]$, i.e., the limit $L \rightarrow \infty$ with $\beta, \ell$ fixed.

For the long interval $B$ we have the EE [24]

$$
S_{B}=\frac{c}{3} \log \left(\frac{\beta}{\pi \epsilon} \sinh \frac{\pi \ell}{\beta}\right)+\frac{\pi c L}{3 \beta}-I\left(1-\mathrm{e}^{-\frac{2 \pi \ell}{\beta}}\right) .
$$

The function $I(x)$ is the mutual information of two intervals on a complex plane with cross ratio $x$. The small $x$ expansion of $I(x)$ to order $x^{8}$ was calculated in Refs. [8,29] and to order $x^{10}$ was calculated in Refs. [30,31]. Note that nothing but tediousness prevents one from calculating the mutual information to even higher orders of $\ell$. Combining with the fact that $S_{B, i}=S_{A, i}$, we obtain the long interval Holevo information

$$
\begin{aligned}
\chi_{B}= & \frac{\pi c L}{3 \beta}-\frac{2 \pi^{3}(4 \pi L-7 \beta) \ell^{4}}{315 \beta^{4} L}+\frac{32 \pi^{5} \ell^{5}}{3465 \beta^{5}} \\
& +\frac{8 \pi^{4}\left(32 \pi^{2} L^{2}-143 \pi \beta L\right) \ell^{6}}{135135 \beta^{6} L^{2}}+\cdots+O\left(\ell^{11}, 1 / c\right) .
\end{aligned}
$$

One can find full form of the equation in (S18) of the Supplemental Material [25]. Note that $S(L)-\chi_{B}$ is nonvanishing in the thermodynamic limit.

We denote the results of Eqs. (14) and (16) as the CFT Holevo information $\chi_{\mathrm{CFT}}(\ell)$ and $\chi_{\mathrm{CFT}}(L-\ell)$, respectively. Note that they are only valid for $\ell \ll \beta \ll L$. They are consistent with holographic Holevo information $\chi_{\text {holo }}$ [Eq. (11)] at the leading order of large $c$, while at the subleading orders we see the corrections. We plot them in Fig. 1. We see that, with $1 / c$ corrections, both the short and long interval plateaus are resolved.

The leading $c$ of Eq. (13) is consistent with the result

$$
\sum_{i} p_{i} S_{A, i}=\frac{c}{3} \log \left(\frac{\beta}{\pi \epsilon} \sinh \frac{\pi \ell}{\beta}\right)+O\left(c^{0}\right),
$$

which was gotten in Ref. [2] by assuming that the contributions from the primary excited states dominate the average. In fact, from the result in Ref. [32], we can show that there are far more descendant states than primary states in high levels of a large $c$ CFT [26]. It is intriguing to show explicitly why primary excited states dominate the average. Supposing that Eq. (17) is valid as long as $\ell<L / 2$, one can get Holevo information by Bao and Ooguri in Ref. [2] with the following:

$\chi_{\mathrm{BO}}(\ell)= \begin{cases}0 & \ell<L / 2 \\ \frac{c}{3} \log \frac{\sinh \frac{\pi \ell}{\beta}}{\sinh \frac{\pi(L-\ell)}{\beta}} & L / 2<\ell<L-\frac{\beta}{2 \pi} \log 2, \\ \frac{\pi c L}{3 \beta} & \ell>L-\frac{\beta}{2 \pi} \log 2\end{cases}$ 
It is a combination of the holographic and CFT results, and it is the leading order $c$ Holevo information. For comparison, we also plot $\chi_{\mathrm{BO}}$ in Fig. 1.

Canonical ensemble thermal state with low temperature.-In low temperature limit, we have $\beta \gg L$. The dual gravity background is the thermal AdS and the holographic thermal entropy is vanishing

$$
S_{\text {holo }}(L)=0 \text {. }
$$

From $0 \leq \chi(\ell) \leq S(L)$, we obtain

$$
\chi_{\text {holo }}(\ell)=0 .
$$

In CFT, the above total indistinguishability can be lifted by taking into account the finite-size effect exponentially suppressed by the factor $q=\mathrm{e}^{-2 \pi \beta / L}$. Using the results in Ref. [24] and considering only the contributions from the holomorphic sector of the vacuum conformal family, for the short interval we get

$$
\begin{aligned}
\chi_{A}= & \left(\frac{32 q^{2}}{15 c}+\frac{24 q^{3}}{5 c}+\frac{64 q^{4}}{5 c}+O\left(q^{5}\right)\right)\left(\frac{\pi \ell}{L}\right)^{4} \\
& +\left(\frac{128(c-16) q^{2}}{315 c^{2}}+\frac{32(c-24) q^{3}}{35 c^{2}}\right. \\
& \left.+\frac{256(c-40) q^{4}}{105 c^{2}}+O\left(q^{5}\right)\right)\left(\frac{\pi \ell}{L}\right)^{6}+O\left(\ell^{8}\right),
\end{aligned}
$$

and for the long interval we obtain

$$
\begin{aligned}
\chi_{B}-S(L)= & -\left[\frac{32 \pi \beta\left(\beta^{2}+L^{2}\right)\left(4 \beta^{2}+L^{2}\right)}{15 L^{5}} q^{2}+O\left(q^{3}\right)\right] \\
& \times\left(\frac{\pi \ell}{L}\right)^{4}+O\left(\ell^{5}\right) .
\end{aligned}
$$

Microcanonical ensemble thermal state.-We now consider the microcanonical ensemble thermal state with fixed high energy $E$, with contributions from both the holomorphic and antiholomorphic sectors. We have the thermal state [Eq. (1)] with

$$
p_{i}=\frac{\delta\left(E-E_{i}\right)}{\Omega(E)} .
$$

At energy $E$ the number of states $\Omega(E)$ is given by the Cardy formula [33] and it is an inverse Laplace transformation of canonical ensemble partition function $Z(\beta)$. Beyond the saddle point approximation of Refs. [33,34], it turns out that

$$
\Omega(E)=\sqrt{\frac{\pi c L}{6 E}} I_{1}\left(\sqrt{\frac{2 \pi c L E}{3}}\right),
$$

with $I_{\nu}$ being a modified Bessel function of the first kind. As with the case of the canonical ensemble thermal state with high temperature, we omit the exponentially suppressed terms of large $E$ but keep the power suppressed terms.

The Cardy formula can be generalized to the cases of various multipoint correlation functions on a torus [32,35-37], i.e., in a canonical ensemble thermal state. One can use the inverse Laplace transformation of the canonical ensemble average to obtain the corresponding microcanonical ensemble one. In this way, we can derive the one-point functions, and thus the short interval EE, of the microcanonical ensemble thermal state from the canonical ensemble one-point functions. Similarly, we can obtain the microcanonical ensemble average short interval EE from the corresponding canonical ensemble one. Combining the short interval EE and average EE, we obtain Holevo information:

$\chi_{A}=\frac{\pi^{3} \ell^{4}\left[\pi c L\left(I_{3}-I_{1}\right)+24 \lambda I_{2}\right]}{540 \lambda^{4} L I_{1}}+\cdots+O\left(e^{12}\right)$,

with the definition $\lambda:=\sqrt{(\pi c L / 6 E)}$, which is fixed in the thermodynamic limit, and $I_{\nu}$ being the shorthand notation of $I_{\nu}(\pi c L / 3 \lambda)$. The full form of the equation is presented in (S38) of the Supplemental Material [25].

For the long interval case, we use the OPE of twist operators in Refs. [22-24] and obtain the following result,

$$
\chi_{B}-S(L)=O\left(\ell^{12}\right) .
$$

However, we cannot get the term of order $\ell^{12}$ explicitly. It is possibly nonvanishing. See details in the Supplemental Material [25].

Contribution from a nonidentity primary operator.Lastly, we consider the leading contribution to Holevo information from a nonidentity primary operator $\psi$ with normalization $\alpha_{\psi}$, conformal weights $\left(h_{\psi}, \bar{h}_{\psi}\right)$. We have the scaling dimension $\Delta_{\psi}=h_{\psi}+\bar{h}_{\psi}$ and spin $s_{\psi}=h_{\psi}-\bar{h}_{\psi}$. For a general thermal state with density matrix [Eq. (1)], we use the OPE of twist operators [7,16-24] and get the short and long interval Holevo information

$$
\begin{aligned}
\delta_{\psi} \chi_{A}= & \frac{\sqrt{\pi} \Gamma\left(\Delta_{\psi}+1\right) \ell^{2 \Delta_{\psi}}}{2^{2 \Delta_{\psi}+2} \Gamma\left(\Delta_{\psi}+\frac{3}{2}\right)} \frac{\mathrm{s}_{\psi}}{\alpha_{\psi}} \\
& \times\left[\sum_{i} p_{i}\langle\psi\rangle_{\rho_{i}}^{2}-\left(\sum_{i} p_{i}\langle\psi\rangle_{\rho_{i}}\right)^{2}\right]+o\left(\ell^{2 \Delta_{\psi}}\right), \\
\delta_{\psi} \chi_{B}= & \delta_{\psi} S(L)-\frac{\ell^{2 \Delta_{\psi}}}{2^{2 \Delta_{\psi}}+1} \frac{\mathrm{i}^{2 s_{\psi}}}{\alpha_{\psi}} \sum_{i \neq i^{\prime}}\left\langle i|\psi| i^{\prime}\right\rangle\left\langle i^{\prime}|\psi| i\right\rangle p_{i} \partial_{n} \\
& \times\left[\sum_{j=1}^{n-1} \frac{\left(p_{i^{\prime}} / p_{i}\right)^{j}}{\left(\sin \frac{\pi j}{n}\right)^{2 \Delta_{\psi}}}\right]_{n=1}+o\left(\ell^{2 \Delta_{\psi}}\right) .
\end{aligned}
$$

These forms are general and can be applied to both canonical ensemble and microcanonical ensemble thermal states. 
The results however are not universal in the sense that they depend on the structure constants, so we cannot evaluate their explicit forms without knowing the details of the theory. See more details in the Supplemental Material [25].

Discussion.-For the conclusion of this Letter, we would like to mention the implication of the almost vanishing short interval Holevo information to our recent finding of nongeometric states in Ref. [11]. As shown in Ref. [11] some special descendant states are nongeometric, which indicates that they cannot be locally thermal. The ensemble average for obtaining the Holevo information is over all states including those nongeometric descendant states. However, we see the resultant leading order $c$ short interval Holevo information is still consistent with thermality. Using the results in Ref. [32] we can show that there are far more descendant states than primary ones at high levels in a large $c$ CFT [26]. This indicates that the contributions from the nongeometric descendant states are suppressed. It is intriguing to show how this happens explicitly.

We would like thank Alice Bernamonti, Pasquale Calabrese, Federico Galli, Manuela Kulaxizi, Hong Liu, Andrei Parnachev, Tadashi Takayanagi, and Erik Tonni for helpful discussions. J.Z. would like to thank the Galileo Galilei Institute for Theoretical Physics and the organizers of the workshop "Entanglement in Quantum Systems" for hospitality and for being given the opportunity to present part of the result of work, and to thank participants of the workshop for helpful discussions. W. Z. G. is supported in part by the National Center of Theoretical Science (NCTS). F. L. L. is supported by Taiwan Ministry of Science and Technology through Grant No. 103-2112-M-003-001-MY3. J. Z. is supported in part by Italian Ministero dell'Istruzione, Università e Ricerca (MIUR), and Istituto Nazionale di Fisica Nucleare (INFN) through the "Gauge Theories, Strings, Supergravity" (GSS) research, and by Fondazione Cariplo and Regione Lombardia, Grant No. 2015-1253.

¥wzguo@cts.nthu.edu.tw

*Corresponding author.

linfengli@phy.ntnu.edu.tw

${ }^{\dagger}$ Corresponding author. jiaju.zhang@unimib.it

[1] J. M. Maldacena, Adv. Theor. Math. Phys. 2, 231 (1998); Int. J. Theor. Phys. 38, 1113 (1999).

[2] N. Bao and H. Ooguri, Phys. Rev. D 96, 066017 (2017).

[3] M. Bañados, C. Teitelboim, and J. Zanelli, Phys. Rev. Lett. 69, 1849 (1992).

[4] J. D. Brown and M. Henneaux, Commun. Math. Phys. 104, 207 (1986).

[5] S. Ryu and T. Takayanagi, Phys. Rev. Lett. 96, 181602 (2006).
[6] V. E. Hubeny, M. Rangamani, and T. Takayanagi, J. High Energy Phys. 07 (2007) 062.

[7] M. Headrick, Phys. Rev. D 82, 126010 (2010).

[8] T. Barrella, X. Dong, S. A. Hartnoll, and V. L. Martin, J. High Energy Phys. 09 (2013) 109.

[9] T. Faulkner, A. Lewkowycz, and J. Maldacena, J. High Energy Phys. 11 (2013) 074.

[10] B. Michel and A. Puhm, J. High Energy Phys. 07 (2018) 179.

[11] W.-Z. Guo, F.-L. Lin, and J. Zhang, arXiv:1806.07595.

[12] B. Chen, J.-B. Wu, and J.-j. Zhang, J. High Energy Phys. 08 (2016) 130.

[13] F.-L. Lin, H. Wang, and J.-j. Zhang, J. High Energy Phys. 11 (2016) 116.

[14] S. He, F.-L. Lin, and J.-j. Zhang, J. High Energy Phys. 08 (2017) 126.

[15] S. He, F.-L. Lin, and J.-j. Zhang, J. High Energy Phys. 12 (2017) 073.

[16] P. Calabrese and J. L. Cardy, J. Stat. Mech. (2004) P06002.

[17] J. L. Cardy, O. A. Castro-Alvaredo, and B. Doyon, J. Stat. Phys. 130, 129 (2008).

[18] P. Calabrese, J. Cardy, and E. Tonni, J. Stat. Mech. (2011) P01021.

[19] M. Rajabpour and F. Gliozzi, J. Stat. Mech. (2012) P02016.

[20] B. Chen and J.-j. Zhang, J. High Energy Phys. 11 (2013) 164.

[21] D. Bianchini, O. A. Castro-Alvaredo, and B. Doyon, Nucl. Phys. B896, 835 (2015).

[22] B. Chen and J.-q. Wu, Phys. Rev. D 91, 086012 (2015).

[23] B. Chen and J.-q. Wu, Phys. Rev. D 92, 106001 (2015).

[24] B. Chen, Z. Li, and J.-j. Zhang, J. High Energy Phys. 09 (2017) 151.

[25] See Supplemental Material at http://link.aps.org/ supplemental/10.1103/PhysRevLett.121.251603 for the full forms of the equations with omitted parts denoted by $\cdots$ in this Letter. In the Supplemental Material one can also find more calculation details.

[26] W.-z. Guo, F.-L. Lin, and J. Zhang, arXiv:1810.01258.

[27] N. Lashkari, A. Dymarsky, and H. Liu, J. Stat. Mech. (2018) 033101.

[28] A. Dymarsky, N. Lashkari, and H. Liu, Phys. Rev. E 97, 012140 (2018).

[29] B. Chen, J. Long, and J.-j. Zhang, J. High Energy Phys. 04 (2014) 041.

[30] M. Beccaria and G. Macorini, J. High Energy Phys. 04 (2014) 045.

[31] Z. Li and J.-j. Zhang, J. High Energy Phys. 05 (2016) 130.

[32] P. Kraus and A. Maloney, J. High Energy Phys. 05 (2017) 160.

[33] J. L. Cardy, Nucl. Phys. B270, 186 (1986).

[34] S. Carlip, Classical Quantum Gravity 17, 4175 (2000).

[35] E. M. Brehm, D. Das, and S. Datta, arXiv:1804.07924.

[36] A. Romero-Bermúdez, P. Sabella-Garnier, and K. Schalm, J. High Energy Phys. 09 (2018) 005.

[37] Y. Hikida, Y. Kusuki, and T. Takayanagi, Phys. Rev. D 98, 026003 (2018). 